\title{
LA EXPLOTACIÓN DE LAS PLANTAS Y LOS INICIOS DE LA AGRICULTURA EN EL PRÓXIMO ORIENTE: 20 AÑOS DE INVESTIGACIÓN ARQUEOBOTÁNICA
}

\author{
Leonor Peña-Chocarro (Instituto de Historia, CSIC) \\ Amaia Arranz-Otaegui (Universidad de Copenhague)
}

\begin{abstract}
RESUMEN
Este trabajo aborda el desarrollo de la investigación arqueobotánica en el Próximo Oriente en las dos últimas décadas. El artículo se centra en los avances conseguidos en el estudio de la explotación de las plantas en periodos pre-agrarios así como en los orígenes de la agricultura. Se discuten los principales yacimientos paleolíticos y epipaleolíticos en los que se documenta el uso de las plantas, y se abordan temas como el cultivo pre-doméstico y la domesticación de las plantas.
\end{abstract}

\section{PALABRAS CLAVE}

arqueobotánica, orígenes agricultura, epipaleolítico, neolítico, plantas silvestres, plantas domésticas

\section{SUMMARY}

This paper deals with the development of Near Eastern archaeobotany during the past two decades. It focuses on the advances of the discipline, particularly on the progress made in pre-agrarian plant use and the origins of agriculture. The main Palaeolithic and Epipalaeolithic sites where plant use has been well attested are discussed while issues such as pre-domestic cultivation and plant domestication are also analyzed.

\section{KEYWORDS}

Archaeobotany, origins of agriculture, Epipaleolithic, Neolithic, wild plants, domesticated plants

\section{Introducción}

El origen de la agricultura en el Próximo Oriente es uno de los temas que más interés ha suscitado tradicionalmente entre investigadores y estudiosos de la Prehistoria. Se trata de uno de los acontecimientos más fascinantes de la larga historia de la especie humana que transformó radicalmente la forma de vida que los seres humanos habían mantenido durante milenios. Este fenómeno, marca la transición de una subsistencia basada en la explotación de recursos silvestres a otra en la que la agricultura irá paulatinamente ocupando un lugar predominante en la obtención de recursos. El esfuerzo por conocer los detalles de este proceso, ha generado una importante cantidad de trabajos, que en los últimos 20 años se ha incrementado de forma considerable gracias a la aplicación de nuevas técnicas y desarrollos metodológicos.

La investigación sobre los orígenes de la agricultura no es ciertamente un tema reciente; ya desde mediados del siglo XX diferentes investigadores dedicaron ingentes esfuerzos a estudiar la domesticación y explotación de las plantas durante el neolítico en el Próximo Oriente (Braidwood, 1960; Dimbleby, 1978; Higgs and Jarman, 1969; Ucko and Dimbleby, 1969), proponiendo explicaciones en base a la disponibilidad de los datos en aquel momento. Yacimientos del suroeste asiático tan importantes como Jarmo, Jericho, Çatal Höyük, Haçilar o Beidha, proporcionaron restos carbonizados de cereales domésticos que contribuyeron al conocimiento de los inicios de la producción de alimento. Es a partir de la década de los 70, con la implantación de la flotación como método para la recuperación de restos vegetales, cuando el corpus de datos arqueobotánicos se incrementa como demuestra la bibliografía especializada. Los detallados trabajos de Willem van Zeist 
(van Zeist, 1986; Van Zeist and Bakker-Heeres, 1982, 1984; van Zeist and de Roller, 1995, 2003) o la investigación tanto arqueobotánica (Hillman, 1978, 1996; Hillman et al., 1989; McLaren et al., 1990; Moore and Hillman, 1992; Moore et al., 2000) como etnográfica (Hillman, 1981, 1984, 1985) del recientemente fallecido Gordon Hillman constituyen piezas fundamentales de la investigación de las últimas décadas del siglo XX.

El siglo XXI se inaugura con una profusión de trabajos centrados en diferentes líneas de investigación. Además de los análisis puramente arqueobotánicos de numerosos yacimientos, muchos estudios se han centrado en delimitar la zona o zonas en la que se produce la domesticación de los cereales y su cronología (Abbo et al., 2010; Allaby et al., 2010; Fuller et al., 2011; Heun et al., 1997; Ibáñez et al., 2018; Lev-Yadun et al., 2000; Purugganan and Fuller, 2011), así como en explorar y caracterizar el desarrollo y las causas de este proceso. Así pues, se ha trabajado por conocer mejor las especies involucradas y su domesticación (Arranz-Otaegui et al., 2018b; Caracuta et al., 2016; Fuller et al., 2011; Tanno and Willcox, 2006b, 2012; Weiss and Zohary, 2011), la variabilidad regional (Arranz-Otaegui et al., 2016b; Douché and Willcox, 2018; Weide et al., 2018; Whitlam et al., 2018), el cultivo pre-doméstico (Colledge and Conolly, 2010; Weiss et al., 2006; White and Makarewicz, 2012; Willcox et al., 2008, 2009), las prácticas agrícolas o la tecnología (Arranz-Otaegui et al., 2016b; Eitam et al., 2015; Ibáñez et al., 2018) entre otros muchos aspectos. También, se han incrementado los trabajos centrados en la explotación de las plantas por parte de los grupos cazadores-recolectores demostrando que, con anterioridad a la domesticación de las plantas y la aparición de la agricultura, existió un largo periodo durante el cual se produjo una importante intensificación en la explotación y control de las plantas.

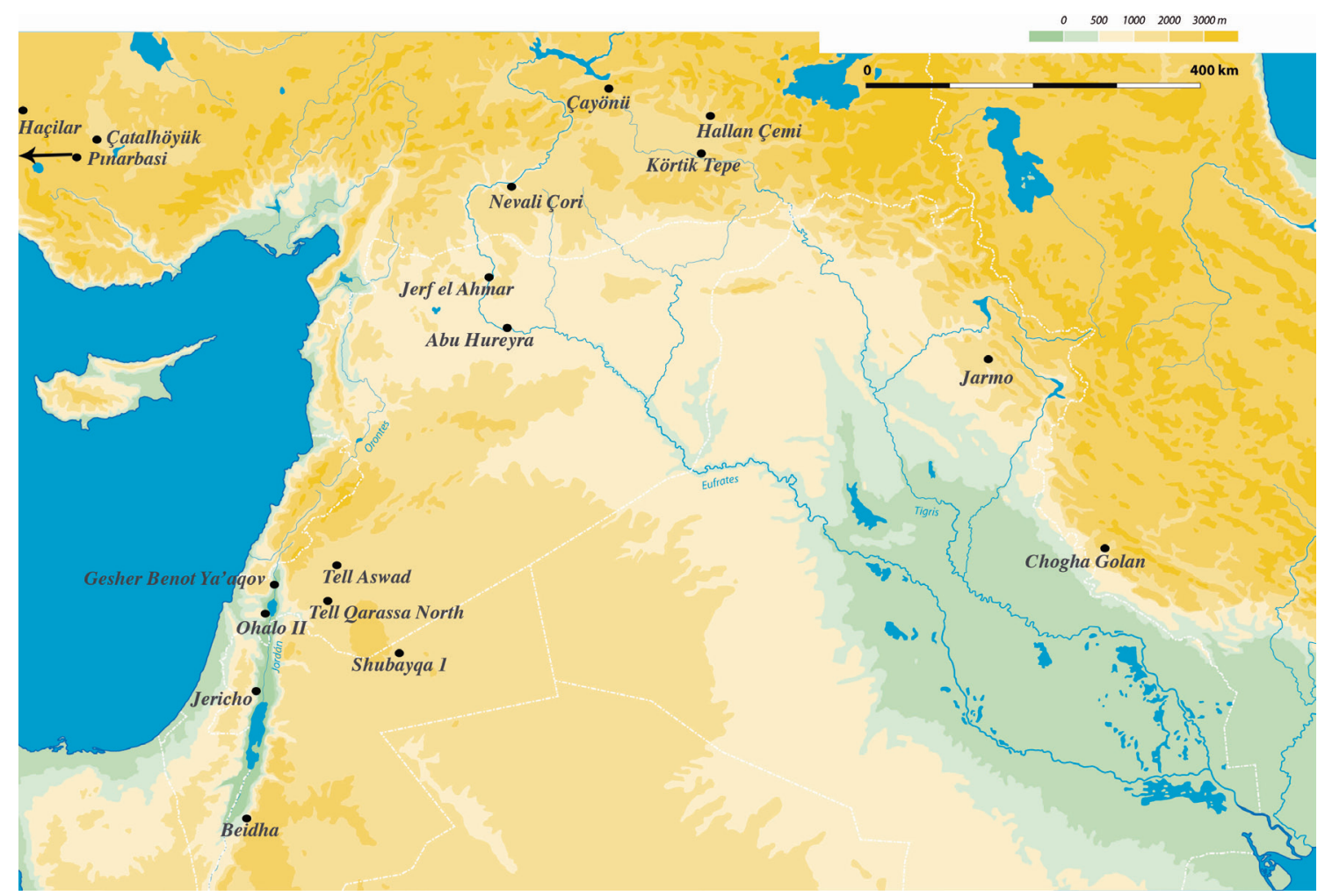

Figura 1. Mapa con los principales yacimientos Epipaleolíticos y Neoliticos Pre-Cerámicos en el Próximo Oriente (mapa en blanco cedido por Luis Teira) citados en el texto.

En este trabajo nos centraremos en los avances que se han producido en las dos últimas décadas en el estudio de la utilización de las plantas por parte de las comunidades prehistóricas del Próximo Oriente. Primero abordaremos la explotación de las plantas 
entre los grupos cazadores-recolectores, como ejemplo del importante papel de las plantas antes de la implantación de la agricultura. A continuación, haremos una valoración de la información sobre el cultivo pre-doméstico como preludio de la agricultura, y, finalmente nos centraremos en la transformación del conocimiento sobre los tiempos y los lugares de la domesticación, y por tanto de los orígenes de la agricultura. Se trata, pues, de presentar los avances de la investigación en torno a la explotación de las plantas y el origen de la agricultura, conseguidos en los últimos 20 años, siguiendo un orden cronológico.

\section{La explotación de las plantas entre los cazadores-recolectores del Paleolítico y Epipaleolítico}

La explotación de los recursos vegetales silvestres por parte de las comunidades paleolíticas (Goren-Inbar et al., 2002; Melamed et al., 2016; Nadel et al., 2013; Weiss, 2017; Weiss et al., 2004a) y epipaleolíticas (Arranz-Otaegui et al., 2018a , 2018b; Savard et al., 2006; Weiss et al., 2004a, 2004b, 2008) de esta región ha sido un tema recurrente en los últimos años.

A pesar de la baja densidad de restos, y de las diferencias tan evidentes en la disponibilidad de restos en comparación con periodos posteriores, durante el Paleolítico Superior y el Epipaleolítico, también se constata la utilización de especies vegetales y su conservación en el registro arqueológico. Las diferencias entre la disponibilidad de restos vegetales entre unos periodos y otros están en relación no sólo al grado de preservación sino también a cuestiones relacionadas con el procesado de las especies vegetales que favorecerán en mayor o menor grado la conservación de los restos.

En este breve espacio, citaremos tres ejemplos, que constituyen quizás la evidencia más significativa de la utilización de plantas en el Paleolítico del Próximo Oriente. Por su antigüedad, destacamos el estudio de los materiales arqueobotánicos del yacimiento achelense de Gesher Benot Ya'aqov en Israel (Melamed et al., 2016) en el que se documentaron 117 taxones vegetales (semillas, frutos, frutos secos, verdura y tubérculos), de los cuales 55 corresponden a plantas comestibles con una cronología de 780.000 años. Los más abundantes en el registro arqueobotánico, y más nutritivos, son los frutos secos y los tubérculos, seguidos por la fruta. Se documentan además algunas especies extinguidas en la zona. En general, se constata una gran diversidad de especies que combinadas proporcionarían una alimentación muy nutritiva, rica en hidratos de carbono, proteínas, almidón y grasas. El estudio, por tanto, enfatiza la diversidad de la dieta vegetal de estos grupos humanos y demuestra el conocimiento humano del entorno natural. Es evidente que la recolección por parte de los homínidos de esta amplia variedad de especies requiere un conocimiento de las zonas en las que las plantas crecieron, de su toxicidad, así como de sus patrones de estacionalidad, etc. Por otra parte, el manejo del fuego, bien documentado en el yacimiento (Goren-Inbar et al., 2004), debió contribuir a mejorar la palatabilidad y digestibilidad de algunas de las especies documentadas. Este yacimiento es, por lo tanto, un ejemplo clave para la comprensión de la gran variedad de recursos utilizados por estos grupos de homínidos, que a través del corredor levantino, abandonaron el continente africano a la conquista de Eurasia.

Otro interesante ejemplo de este periodo es el yacimiento de Ohalo II (Nadel, 2002), situado a orillas del Lago Kinneret, en Israel, que proporcionó un numeroso conjunto de restos vegetales carbonizados de gran antigüedad (ca. 23.000 BP). La abundancia de restos dio lugar a un detallado estudio detallado sobre la utilización de las plantas por en este campamento. El trabajo pionero de Dolores Piperno (Piperno et al., 2004), basado en el análisis de almidones de uno los molinos del yacimiento, proporcionó la evidencia más antigua del uso de cereales silvestres y puso de relieve el potencial del estudio de microrrestos vegetales (hecho que en las siguientes décadas se tradujo en un incremento 
substancial de publicaciones sobre almidones y fitolitos). En cuanto a macrorrestos, se identificaron más de 90.000 restos adscritos a 142 taxones diferentes, de los cuales al menos 30 se corresponden con plantas de uso económico (Weiss et al., 2004b). El estudio puso de manifiesto la importancia de las gramíneas que incluye alrededor de 19.000 restos pertenecientes a varias especies. Entre ellas, predominaban las semillas de tamaño pequeño (small seeded grasses), aunque también se documentaron cereales silvestres como la cebada silvestre (Hordeum spontaneum), o una especie de trigo silvestre (Triticum dicoccoides) que constituyeron una parte importante de la dieta vegetal. También se identificaron otras especies de importancia como las bellotas (Quercus), almendras (Amygdalus), pistachos (Pistacia), aceitunas silvestres (Olea), leguminosas silvestres, así como higos (Ficus), uvas silvestres (Vitis), etc. Los diferentes estudios que se han llevado a cabo en el yacimiento sugieren una ocupación a lo largo de todo el año, así como una economía de amplio espectro basada en la explotación de recursos animales (mamíferos, aves, peces) y vegetales que resulta anterior a lo que se presuponía.

Recientemente, se ha publicado (por una de las autoras de este trabajo, AA-O) los resultados del estudio arqueobotánico del yacimiento jordano de Shubayqa (14,6-11,6 ka cal. BP.) que ha proporcionado un conjunto de más de 65.000 macrorrestos vegetales pertenecientes a 95 taxones (Arranz-Otaegui et al., 2018a) entre los que destaca la presencia de tubérculos, leguminosas y algunos cereales silvestres. Junto a ellos, se ha identificado un número importante de fragmentos carbonizados de comida, interpretados como fragmentos de pan, cuyos ingredientes fundamentales son los cereales mezclados con restos de tubérculos, muy probablemente de Bolboschoenus glaucus, una planta de la misma familia que la chufa, cuyos restos son muy frecuentes en el yacimiento.

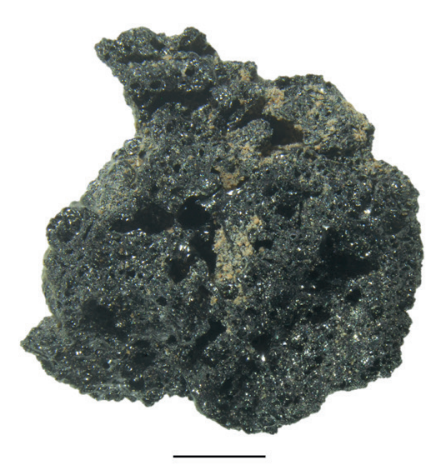

Figura 2a. Restos de pan del yacimiento Shubayqa 1, escala $1 \mathrm{~mm}$., identificados por L. González-Carretero (foto Amaia ArranzOtaegui).

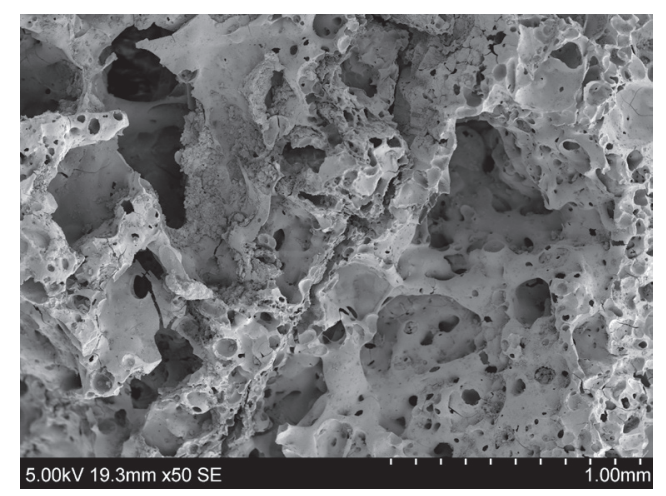

Figura $2 b$. Detalle de fragmento de pan con microscopio electrónico de barrido (foto L. González-Carretero y A. Arranz-Otaegui).

Este descubrimiento sitúa la aparición de pan 4000 años antes de que la agricultura se iniciara en el Próximo Oriente y supone un importante paso adelante en el conocimiento de las prácticas de subsistencia de los cazadores-recolectores epipaleolíticos. Por otra parte, demuestra que a los macrorrestos vegetales habitualmente estudiados (semillas y frutos) hay que añadir, y explorar en el futuro, los tubérculos y otros órganos subterráneos que, sin duda, como este estudio demuestra, debieron jugar un papel muy importante en la dieta vegetal de los cazadores-recolectores.

\section{Los preludios de la domesticación: el cultivo pre-doméstico}

La domesticación de las plantas constituye uno de los aspectos clave en el debate sobre los orígenes de la agricultura. Se trata de un término con diferentes significados en los varios ámbitos disciplinarios. En nuestro ámbito, la domesticación se refiere a la 
selección de una serie de rasgos en los cultivares que afectan fundamentalmente, pero no sólo, a la pérdida de los mecanismos de reproducción natural de la planta (Willcox 2012). Una vez domesticada, la planta pasa a depender del control humano para sobrevivir. La domesticación tuvo lugar después de un largo periodo de intensificación en el uso de las plantas, que incluyó el cultivo de cereales silvestres. Además de la pérdida de la capacidad pata reproducirse, la domesticación también conlleva un aumento del tamaño del grano, se pierde la sensibilidad de la planta a los cambios ambientales a la hora de germinar y florecer, y se produce una homogeneización en la maduración de las espigas.

La domesticación conlleva una serie de modificaciones morfológicas en la planta que son identificables en los restos arqueobotánicos que permiten identificar la presencia de ejemplares cultivados y silvestres. En el caso particular de los cereales, las poblaciones silvestres (gramíneas silvestres) poseen un raquis frágil que, al llegar la planta a la madurez, se rompe provocando la desarticulación de la espiga y la dispersión las espiguillas que germinarán un año después. Por el contrario, en las poblaciones domésticas (los cereales), el raquis es morfológicamente diferentes, más duro y sólido, y no se rompe al llegar la planta a su punto de madurez, manteniéndose la espiga entera y evitando que esta se desarticule y se produzca la dispersión de sus semillas. Es precisamente, el tipo de cicatriz que queda cuando el raquis se rompe, el elemento utilizado avala la presencia de plantas silvestres o domesticadas. Sin embargo, la sola presencia de raquis de tipo sólido no es garantía de domesticación ya que, de forma natutal, este tipo de raquis puede aparecer en algunos mutantes en poblaciones silvestres, aunque en muy pequeñas proporciones. Por lo tanto, es imprescindible cuantificar los diferentes tipos de raquis y evaluar las proporciones de los tipos domésticos y silvestres.

Durante las dos últimas décadas los datos sobre la explotación de las plantas durante el Paleolítico Superior y, especialmente, durante el Epipaleolítico, han aumentado de forma exponencial, demostrando el uso intensivo de muchas especies vegetales entre las que se encuentran los antecesores silvestres de los primeros cereales domésticos. La domesticación de las plantas puede ser considerada, por lo tanto, como el resultado de un proceso evolutivo a partir del cultivo sistemático de cereales y leguminosas morfológicamente silvestres que daría lugar en un lapso de 1000-2000 años a la aparición de la agricultura (Arranz-Otaegui et al., 2016b). La evidencia arqueobotánica para la zona levantina alrededor de 11,5 ka cal BP durante el Neolítico Pre-Cerámico A (PPNA) sugiere que diferentes cereales silvestres como el trigo y la cebada se están cultivando en este momento (Colledge, 2001; Willcox et al., 2008). Se trata de especies silvestres, aún no domesticadas, pero que se cultivan. Un milenio más tarde (ca. 10,5 ka cal BP), durante el inicio del Neolítico Pre-Cerámico B (EPPNB), aparecen en el registro arqueobotánico las primeras evidencias de cereales morfológicamente domésticos (es decir, aquellos que presentan raquis sólidos y resistentes en proporciones superiores al 10-20\%). La agricultura, entendida ésta como un sistema económico basado en el cultivo de especies domésticas, se desarrolla más tarde, alrededor de 9,8 ka cal BP, durante el Neolítico Pre-cerámico B medio y tardío (M/LPPNB).

En la década de los 90 y 2000, el yacimiento de Abu Hureyra (Siria) marcó un importante hito al interpretarse (Hillman et al., 2001) que los grupos natufienses (13.000 BP) cultivaron cereales. De hecho, se sostenía que el centeno era una de las especies cultivadas por los grupos de cazadores-recolectores de este yacimiento. Sin embargo, trabajos posteriores (Colledge and Conolly, 2014) reinterpretaron la evidencia y demostraron que en este yacimiento los cereales silvestres están muy poco representados, predominando las plantas silvestres en el registro arqueobotánico.

Determinar el cultivo de cereales silvestres es uno de los aspectos en los que la investigación ha avanzado considerablemente en estas dos últimas décadas. Así pues, se han establecido una serie de parámetros (Willcox et al., 2008; Willcox, 2012) que permiten aproximarse a esta práctica en conjuntos arqueobotánicos pre-agrarios. En cualquier caso, 
es necesario considerar varios de estos parámetros conjuntamente. En primer lugar, la presencia de granos de cereal con morfologías y tamaños más cercanos a los de los cereales domésticos es un indicador de posible cultivo, así como el aumento del tamaño de los granos a medida que se avanza en el tiempo. La presencia de especies fuera de su zona de hábitat natural es también un elemento a tener en cuenta a la hora de evaluar la presencia de cultivo pre-doméstico. También, la existencia de plantas adventicias (malas hierbas) características de campos de cultivo junto a los cereales, es un buen marcador.

La investigación desarrollada en el Próximo Oriente ha evidenciado el cultivo predoméstico en varios yacimientos del neolítico Pre-cerámico A (PPNA) tanto en la zona meridional y central de la región levantina, en el Eúfrates y en la región de los montes Zagros, aunque los datos disponibles son muy variados dependiendo de las regiones (Arranz-Otaegui et al., 2016b). Así, por ejemplo, en la zona siria la cebada silvestre (Hordeum spontaneum) parece dominar los conjuntos arqueobotánicos, mientras que en el Eúfrates en yacimientos como Jerf el Ahmar los cereales cultivados incluyen la cebada silvestre y una serie de granos cuya identificación es problemática (escaña o centenoTriticum boeoticum/Secale) (Willcox, 2002). También destacan los trabajos realizados en Irán en yacimientos como Chogha Golan (Riehl et al., 2013). Aunque en un primer lugar los datos se interpretaran como un caso excepcional de cultivo-pre doméstico de cereales fuera del Levante (Riehl et al., 2013; Willcox, 2013), los estudios más recientes sugieren que no existió cultivo pre-domestico como tal (Weide et al., 2017). Los datos disponibles indican que en el sureste y centro de Turquía (e.g. Körtik Tepe, Hallan Çemi, Pinarbaşi entre otros) y la zona de los Zagros en Irán, las sociedades neolíticas explotaron otro tipo de recursos vegetales silvestres, que incluían gramíneas como el Aegilops, leguminosas y plantas de la familia de las Cyperaceae y Polygonaceae entre otras muchas (ArranzOtaegui et al., 2016b; Fairbairn et al., 2014; Savard et al., 2006). El trigo y la cebada no se imponen hasta periodos muy posteriores (hace unos 9800 años).

Estos datos sugieren un panorama de diversidad muy complejo en el que parece que las diferentes regiones optaron por conjuntos de especies diferentes que seguramente respondían a las estrategias de subsistencia de cada zona. Todos estos datos apuntan a que, durante la fase más antigua del Neolítico, la dieta vegetal se caracteriza por su gran diversidad, aunque en la zona del Levante se observa que empieza a tomar relevancia un grupo determinado de cereales (el trigo y la cebada).

Por lo que se refiere al Neolítico Pre-Cerámico B (PPNB), los datos sugieren que ya en fechas entre 10.7 y 10.2 cal BP, en yacimientos como Tell Qarassa North (ArranzOtaegui et al., 2016a) se cultivaban varias especies de cereales (cebada, escaña y escanda). El número de raquis pertenecientes a los tipos domésticos parece ser inferior al $50 \%$ por lo que se supone que parte de los cereales presentes en el yacimiento pudieron explotarse a partir de poblaciones silvestres. El registro arqueobotánico de otras zonas del Próximo Oriente apunta a una difusión lenta de los tipos morfológicamente domésticos que no representan más del 10\%, sugiriendo la explotación de especies morfológicamente silvestres en muchos de los yacimientos estudiados (Tanno and Willcox, 2012). El Neolítico Pre-Cerámico B (PPNB) es también el periodo en el que se empieza a constatar la explotación de leguminosas como el garbanzo (Tanno and Willcox, 2006b), el haba (Caracuta et al., 2016) o la lenteja (Willcox et al., 2008). La información disponible sugiere que las leguminosas pudieron haber sido domesticadas durante el PPNB. Sin embargo, se trata de una cuestión todavía abierta a debate debido a que, a diferencia de los cereales, los restos de vainas y otras partes que permiten discriminar entre poblaciones silvestres y domésticas, no son muy habituales en el registro arqueobotánico.

A mediados y finales del PPNB se documentan sociedades propiamente agrícolas como Çatalhöyük (Fairbairn et al., 2002) en las que los cereales y leguminosas domésticos 
constituyen una parte fundamental de la dieta. Un estudio muy reciente sobre restos de comida carbonizados sugiere que, en una primera fase, los cereales se consumirían en forma de pan realizado con harina de cereales, leguminosas y tubérculos (González Carretero et al., 2017). Sin embargo, en una fase posterior, con la integración de la cerámica en la vida cotidiana, parece evidenciarse un cambio en la dieta que conlleva el consumo de nuevos productos a base de cereales como las gachas. También en este periodo, en el Neolítico PreCerámico B medio y antiguo, se observa una mayor explotación de algunas especies, como la escaña (T. monococcum), escanda (Triticum dicoccum), cebada (Hordeum vulgare), lenteja (Lens culinaris), garbanzo (Cicer arietinum), yero (Vicia ervilia) y el guisante (Pisum sativum), considerados como los "cultivos fundadores" de la agricultura neolítica. De todos modos, los estudios arqueobotánicos más recientes indican que las sociedades neolíticas continuaron consumiendo de forma regular plantas silvestres en el marco de una dieta variada (Wallace et al., 2018).

\section{Los lugares y los tiempos de la domesticación de cereales}

"Cuándo, dónde y cómo" surgieron las especies domésticas han sido los tres interrogantes clave en el estudio de los orígenes de la agricultura. Para responder a estas incógnitas los investigadores se han centrado en varios aspectos entre los que resaltamos los intentos por identificar el lugar de origen de la agricultura y precisar el número de domesticaciones (si fue una única domesticación en una región o varios procesos de domesticación en múltiples regiones) que tuvo lugar, el ritmo de desarrollo de la domesticación (rápido o lento) y el tipo de selección (artificial o natural) llevado a cabo.

Tradicionalmente, Anatolia y el sureste de Turquía, han sido considerados como las zonas más probables en las que por primera vez se produjo la domesticación de las plantas (Abbo et al., 2006, 2011; Heun et al., 1997; Lev-Yadun et al., 2000). Durante la década de los 90 e inicios de los 2000, tanto los datos arqueobotánicos como los genéticos apuntaban a esta región como la zona de origen de los llamados "cultivos fundadores" de la agricultura Neolítica. Estos engloban 8 especies: 2 trigos vestidos (la escaña y la escanda), la cebada, 4 leguminosas (lentejas, habas, guisantes y yeros) y el lino (Linum usitatissimum). Yacimientos como Nevali Çori (Pasternak, 1998) y Cayonü (van Zeist and de Roller, 1991/1992, 2003) eran en esos años algunos de los yacimientos mejor estudiados. Datados hacia entre 10.7 y 10,2 ka cal BP, contaban en su registro con la presencia de estos cultivos. Además, los datos genéticos de especies como la escaña (Heun et al., 1997), la escanda (Özkan et al., 2002), la lenteja (Ladizinsky, 1999) o el garbanzo (Ladizinsky and Adler, 1976), indicaban que estas especies se habían domesticado una única vez (origen monofilético) y que las poblaciones silvestres que crecían en sureste de Turquía (en la zona del Karakadag) eran genéticamente las más próximas a las especies domesticadas actuales. Los datos, por tanto, sugerían que esta zona era el lugar de origen de las plantas domésticas, y por ende cuna de las primeras sociedades agrícolas.

El hecho de que fuera una sola zona de domesticación y no varias tenía implicaciones importantes para entender cómo ocurrió el proceso de domesticación ya que se planteaba la hipótesis de que las poblaciones neolíticas del sureste de Turquía habrían seleccionaron los “cultivos fundadores" por sus valores nutritivos (Abbo et al., 2005). Se mantenía que la domesticación fue intencionada, seleccionando de manera artificial aquellas plantas que tenían los rasgos más beneficiosos para el ser humano como semillas de gran tamaño o raquis sólidos que hacían más fácil su recolección. Por otra parte, los experimentos llevados a cabo por Gordon Hillman en la década de los 90 indicaban que, en el caso de los cereales, era posible conseguir poblaciones completamente domésticas en un breve periodo de tiempo que iba de 20 a 200 años (Hillman and Davies, 1990). Así pues, durante los años 90 y buena parte de los 2000 las respuestas a las tres preguntas clave sobre el origen de la 
agricultura fueron relativamente sencillas: la agricultura habría surgido por lo menos hace unos 10,700 años en un área o núcleo central (core area) situado en el sureste de Turquía $\mathrm{y}$, lo habría hecho de manera rápida, gracias a la selección artificial llevada a cabo por los seres humanos. Desde esta zona central, las plantas domésticas se habrían expandido a la zona sur del Levante, a la región de los Zagros y, con posterioridad a Europa.

Ya en la década de los 90 (Harris, 1989, 1996), pero especialmente a partir de los 2000, varios arqueobotánicos y genetistas se mostraron críticos con la hipótesis del área central. Argumentaban que antes de la domesticación debió existir una fase de cultivo predoméstico, dónde las sociedades ya sedentarias comenzaran a experimentar con el cultivo de especies silvestres, pero de manera espontánea. Las primeras evidencias de cultivo pre-domestico aparecen a mediados de la primera década del siglo XXI en yacimientos del Neolítico Pre-Cerámico A (Colledge, 2001; Weiss et al., 2006; Willcox et al., 2008). Además, a partir del año 2006, los análisis arqueobotánicos comenzaron a cuantificar los tipos de raquis y a evaluar la proporción de cereales silvestres y domésticos en cada yacimiento, detalle que aunque siempre se consideró importante, no se había incluido en la mayoría de trabajos arqueobotánicos anteriores a 2010, haciendo imposible discriminar la presencia de plantas silvestres o domésticas de manera eficaz. Este hecho marcó un hito. Por una parte, se observó que la proporción de cereales domésticos incrementaba de manera muy gradual con las primeras evidencias de cultivo de cereales silvestres alrededor del 11,500 BP y la presencia de poblaciones 100\% domésticas entorno al 10,000 BP (según la región). Se ponía de manifiesto, por tanto, que entre ambas fechas habían transcurrido por lo menos 1500 años (Tanno and Willcox, 2006a, 2012). Es decir, la domesticación de cereales no pudo a haber sido un evento único y rápido sino más bien un proceso muy lento y gradual, acorde con una selección de tipo natural (Fuller et al., 2011; Purugganan and Fuller, 2011). Por otra parte, se re-estudiaron yacimientos como Nevali Çori centrándose en la identificación y cuantificación de los raquis silvestres y domésticos y se demostró que no existió una domesticación primigenia en el sureste de Turquía. Es más, las evidencias más antiguas de domesticación de cereales se documentaron en el sur del Levante en yacimientos como Tell Aswad (Tanno and Willcox, 2012) o Tell Qarassa (Arranz-Otaegui et al., 2016a).

Además de los datos arqueobotánicos, los análisis genéticos que se habían utilizado en los años 90 (Amplified Fragment Length Polymorphism o AFLP) que indicaban un único origen de la agricultura en Turquía comenzaron a ser enérgicamente criticados. Genetistas como Allaby y Brown (Allaby and Brown, 2003; Allaby et al., 2008) demostraron que las simulaciones basadas en el AFLP siempre daban un resultado monofilético (un único foco de origen) ya fuera aplicado a plantas de origen monofilético como polifilético (multiples orígenes). Este hecho puso en tela de juicio el valor de la técnica para descifrar los orígenes de los cultivos modernos. Además, análisis genéticos más modernos señalaban que los "cultivos fundadores" podían haberse domesticado más de una vez y en varias regiones del Próximo Oriente (Kilian et al., 2007; Kosterin and Bogdanova, 2008; Molina-Cano, 2005; Mori et al., 2003; Özkan et al., 2011).

Así pues, hoy en día, con los datos disponibles, se puede defender que no existe una evidencia sólida a favor de la hipótesis del núcleo o área central ("core area hypothesis") como la defienden los investigadores Lev-Yadun, Gopher y Abbo (Lev-Yadun et al., 2000). Mucho más plausible es la "hipótesis multi-regional" que sugiere que la domesticación de plantas se produjo en varias regiones más o menos al mismo tiempo. Fue, por lo tanto, un proceso (no un evento) largo y complejo, durante el cual los seres humanos que vivían en diferentes partes del Próximo Oriente, seleccionaron y experimentaron con diversas especies y las cultivaron, y de esa relación simbiótica entre el ser humano y el reino vegetal se produjeron los cambios morfológicos que definen la domesticación. 


\section{Conclusiones}

Como conclusión a este breve artículo sobre los cambios producidos en las dos últimas décadas en el estudio del uso de las plantas y los orígenes de la agricultura en el Próximo Oriente, nos gustaría señalar los grandes avances llevados a cabo en el análisis de nuevas categorías de restos de origen vegetal, como es el caso de los restos de comida carbonizada. Estos nuevos desarrollos metodológicos están permitiendo la identificación de estos restos de alimentos que incluyen, entre otros, fragmentos de pan, tubérculos, etc. que, hasta muy recientemente, pasaban desapercibidos en los conjuntos arqueobotánicos.

Aunque no se han incluido en este trabajo, también se han hecho considerables progresos en el estudio sistemático de fitolitos y almidones (Portillo et al., 2014; Ramsey et al., 2018; Ramsey et al., 2017) que han contribuido a un conocimiento mucho más detallado de la utilización de las plantas por parte de las comunidades prehistóricas. Claramente, las posibilidades que se abren para explorar con más detalle el papel de las plantas en la subsistencia de las comunidades del pasado y comprender mejor las prácticas culinarias de los grupos prehistóricos son enormes y el futuro es prometedor en este sentido.

Además, los avances en los estudios genéticos y la disponibilidad de un mayor número de secuencias estudiadas han permitido reformular las hipótesis sobre los orígenes de la agricultura. Se ha producido un cambio de paradigma y hoy sabemos que con anterioridad a la domesticación de las plantas existió un uso intensivo de las mismas que en determinadas zonas incluyó el cultivo de cereales silvestres. El proceso de domesticación, fue lento y progresivo y, que ocurrió en diferentes lugares del Próximo Oriente de forma simultánea. Por otra parte, también se ha puesto de manifiesto la importancia que pudieron haber tenido otras plantas además de los llamados "cultivos fundadores". La domesticación es, en definitiva, la culminación de un largo proceso en la utilización de las plantas.

Las nuevas investigaciones y los innovadores desarrollos metodológicos permiten vislumbrar un futuro apasionante, que en los próximos años está destinado a proporcionar nuevos e interesantes resultados que enriquecerán nuestro conocimiento sobre este largo proceso de interacción entre las plantas y las comunidades humanas.

\section{Bibliografía}

Abbo, S., Gopher, A., Peleg, Z., Saranga, Y., Fahima, T., Salamini, F., Lev-Yadun, S., 2006, "The ripples of "The Big (agricultural) Bang": the spread of early wheat cultivation", Genome 49 (8), pp. 861-863.

Abbo, S., Gopher, A., Rubin, B., Lev-Yadun, S., 2005, “On the origin of Near Eastern founder crops and the 'dump-heap hypothesis', Genetic Resources and Crop Evolution 52, pp. 491-495.

Abbo, S., Lev-Yadun, S., Gopher, A., 2010, "Agricultural origins: centres and noncentres: a Near Eastern reappraisal”, Critical Reviews in Plant Sciences 29, pp. 317-328.

Abbo, S., Lev-Yadun, S., Gopher, A., 2011, "Origin of Near Eastern plant domestication: homage to Claude Levi-Strauss and "La Pensée Sauvage", Genetic Resources and Crop Evolution 58, pp. 175-179.

Allaby, R., Brown, T., Fuller, D., 2010, "A simulation of the effect of inbreeding on crop domestication genetics with comments on the integration of archaeobotany and genetics: a reply to Honne and Heun", Vegetation History and Archaeobotany 19, pp. 151158.

Allaby, R.G., Brown, T.A., 2003, "AFLP data and the origins of domesticated crops", Genome 46, pp. 448-453.

Allaby, R.G., Fuller, D.Q., Brown, T.A., 2008, "The genetic expectations of a protracted model for the origins of domesticated crops", Proceedings of the National Academy of Sciences 105, pp. 13982-13986. 
Arranz-Otaegui, A., Colledge, S., Ibañez, J.J., Zapata, L., 2016a, "Crop husbandry activities and wild plant gathering, use and consumption at the EPPNB Tell Qarassa North (south Syria)", Vegetation History and Archaeobotany 25, pp. 629-645.

Arranz-Otaegui, A., Colledge, S., Zapata, L., Teira-Mayolini, L.C., Ibáñez, J.J., 2016b, "Regional diversity on the timing for the initial appearance of cereal cultivation and domestication in southwest Asia", Proceedings of the National Academy of Sciences 113, pp. 14001-14006.

Arranz-Otaegui, A., Gonzalez Carretero, L., Ramsey, M.N., Fuller, D.Q., Richter, T., 2018a, "Archaeobotanical evidence reveals the origins of bread 14,400 years ago in northeastern Jordan", Proceedings of the National Academy of Sciences 115, pp. 79257930.

Arranz-Otaegui, A., González Carretero, L., Roe, J., Richter, T., 2018b, "Founder crops" v. wild plants: Assessing the plant-based diet of the last hunter-gatherers in southwest Asia", Quaternary Science Reviews 186, pp. 263-283.

Braidwood, R.J., 1960, “The Agricultural Revolution”, Scientific American 203, pp. $130-152$.

Caracuta, V., Weinstein-Evron, M., Kaufman, D., Yeshurun, R., Silvent, J., Boaretto, E., 2016, "14,000-year-old seeds indicate the Levantine origin of the lost progenitor of faba bean", Scientific Reports 6, pp. 37399.

Colledge, S., 2001, Plant exploitation on Epipalaeolithic and Early Neolithic sites in the Levant. Oxford.

Colledge, S., Conolly, J., 2010, "Reassessing the evidence for the cultivation of wild crops during the Younger Dryas at Tell Abu Hureyra, Syria", Environmental Archaeology 15, pp. 124-138.

Colledge, S., Conolly, J., 2014, "Wild plant use in European Neolithic subsistence economies: a formal assessment of preservation bias in archaeobotanical assemblages and the implications for understanding changes in plant diet breadth", Quaternary Science Reviews 101, pp. 193-206.

Dimbleby, G.W., 1978, Plants and Archaeology. London.

Douché, C., Willcox, G., 2018, "New archaeobotanical data from the Early Neolithic sites of Dja'de el-Mughara and Tell Aswad (Syria): A comparison between the Northern and the Southern Levant", Paléorient 44, pp. 45-57.

Eitam, D., Kislev, M., Karty, A., Bar-Yosef, O., 2015, "Experimental Barley Flour Production in 12,500-Year-Old Rock-Cut Mortars in Southwestern Asia", PLoS ONE 10, e0133306.

Fairbairn, A., Asouti, E., Near, J., Martinoli, D.C., 2002, "Macro-botanical evidence for plant use at Neolithic Çatalhöyük, southcentral Anatolia, Turkey", Vegetation History and Archaeobotany 11, pp. 41-54.

Fairbairn, A.S., Jenkins, E., Baird, D., Jacobsen, G., 2014, "9th millennium plant subsistence in the central Anatolian highlands: new evidence from Pınarbaşı, Karaman Province, central Anatolia", Journal of Archaeological Science 41, pp. 801-812.

Fuller, D.Q., Willcox, G., Allaby, R.G., 2011, "Cultivation and domestication had multiple origins: arguments against the core area hypothesis for the origins of agriculture in the Near East", World Archaeology 43, pp. 628-652.

González Carretero, L., Wollstonecroft, M., Fuller, D., 2017, “A methodological approach to the study of archaeological cereal meals: a case study at Çatalhöyük East (Turkey)", Vegetation History and Archaeobotany 26, pp. 415-432.

Goren-Inbar, N., Alperson, N., Kislev, M.E., Simchoni, O., Melamed, Y., Ben-Nun, A., Werker, E., 2004, "Evidence of hominin control of fire at Gesher Benot Ya'aqov, Israel”, Science 304, pp. 725-727. 
Goren-Inbar, N., Sharon, G., Melamed, Y., Kislev, M., 2002, "Nuts, nut cracking, and pitted stones at Gesher Benot Ya'aqov, Israel", Proceedings of the National Academy of Sciences of the United States of America 99, pp. 2455-2460.

Harris, D.R., 1996, "The origins and spread of agriculture and pastoralism in Eurasia: an overview", in: D.R Harris (ed.), The origins and spread of agriculture and pastoralism in Eurasia, London, pp. 552-573.

Harris, D.R. y Hilmman, G.C, 1989, Foraging and farming. The evolution of plant exploitation. London.

Heun, M., Schäfer-Pregl, R., Klawan, D., Castagna, R., Accerbi, M., Borghi, B., Salamini, F., 1997, "Site of einkorn wheat domestication identified by DNA fingerprinting", Science 278, pp. 1312-1314.

Higgs, E.S., Jarman, M.R., 1969, "The origins of agriculture: a reconsideration”, Antiquity 43, pp. 31-41.

Hillman, G.C., 1978, "On the origins of domestic rye - Secale cereale: The finds from aceramic Can Hasan III in Turkey", Anatolian Studies 28, pp. 157-174.

Hillman, G.C., 1981, "Reconstructing crop husbandry practices from charred remains of crops", in: R. Mercer, R. (ed.), Farming practice in prehistoric Britain. Edinburgh, pp. 123-162.

Hillman, G.C., 1984, "Traditional husbandry and processing of archaic cereals in recent times: the operations, products and equipment which might feature in Sumerian texts. Part I: the glume wheats", Bulletin of Sumerian Agriculture 1, pp. 114-152.

Hillman, G.C., 1985, "Traditional husbandry and processing of archaic cereals in recent times: the operations, products and equipment which might feature in Sumerian texts. Part II: the free-threshing wheats", Bulletin of Sumerian Agriculture 2, pp. 1-31.

Hillman, G.C., 1996, "Late Pleistocene changes in wild plant-foods available to hunter-gatherers of the northern Fertile Crescent: possible preludes to cereal cultivation, in D.R. Harris (ed.), The origins and spread of agriculture and pastoralism in Eurasia, London, pp. 159-203.

Hillman, G.C., Colledge, S.M., Harris, D.R., 1989, "Plant-food economy during the Epipalaeolithic period at Tell Abu Hureyra, Syria: dietary diversity, seasonality, and modes of exploitation, in: D.R. Harris y G.C. Hillman (ed.), Foraging and farming. The evolution of plant exploitation, London, pp. 240-268.

Hillman, G.C., Davies, M.S., 1990, "Measured domestication rates in wild wheats and barley under primitive cultivation, and their archaeological implications", Journal of World Prehistory 4, pp. 157-222.

Hillman, G.C., Davies, M.S., 1992, "Domestication rate in wild wheats and barley under Primitive Cultivation: Preliminary Results and Archaeological Implications of Field Measurements of Selection Coefficient, in: Anderson, P.C. (Ed.), Préhistoire de l'agriculture: nouvelles approches expérimentales et ethnographiques, Paris, pp. 70-102.

Hillman, G.C., Hedges, R.E.M., Moore, A.M.T., Colledge, S., Pettitt, P., 2001, "New evidence of Late glacial cereal cultivation at Abu Hureyra on the Euphrates", The Holocene 11, pp. 383-393.

Ibáñez, J.J., González-Urquijo, J., Teira-Mayolini, L.C., Lazuén, T., 2018, "The emergence of the Neolithic in the Near East: A protracted and multi-regional model", Quaternary International 470, pp. 226-252.

Kilian, B., Ozkan, H., Deusch, O., Effgen, S., Brandolini, A., Kohl, J., Martin, W., Salamini, F., 2007, "Independent wheat B and G genome origins in outcrossing Aegilops progenitor haplotypes", Molecular Biology and Evolution 24, pp. 217-227. 
Kosterin, O.E., Bogdanova, V.S., 2008, "Relationship of wild and cultivated forms of Pisum L. as inferred from an analysis of three markers, of the plastid, mitochondrial and nuclear genomes", Genetic Resources and Crop Evolution 55, pp. 735-755.

Ladizinsky, G., 1999, "Identification of lentil's wild genetic stock", Genetic Resources and Crop Evolution 46, pp. 115-118.

Ladizinsky, G., Adler, A., 1976, "The origin of chickpea Cicer arietinum L.", Euphytica 25, pp. 211-217.

Lev-Yadun, S., Gopher, A., Abbo, S., 2000, “The craddle of agriculture”, Science 288, pp. 1602-1603.

McLaren, F.S., Evans, J., Hillman, G.C., 1990, "Identification of charred seeds from Epipalaeolithic sites of SW Asia", in G.Wagner y E. Perricka (eds.) Proceedings of the 26th International Symposium on Archaeometry, Heidelberg, pp. 787-796.

Melamed, Y., Kislev, M.E., Geffen, E., Lev-Yadun, S., Goren-Inbar, N., 2016, "The plant component of an Acheulian diet at Gesher Benot Ya'aqov, Israel", Proceedings of the National Academy of Sciences 113, pp. 14674-14679.

Molina-Cano, J.L., Russell, J.R., Moralejo, M.A., Escacena, J.L., Arias, G., Powell, W., 2005, "Chloroplast DNA microsatellite analysis supports a polyphphyletic origin for Barley", Theoretical and Applied Genetics 110, pp. 613-619.

Moore, A.M.T., Hillman, G.C., 1992, "The Pleistocene to Holocene transition and human economy in soutwest Asia: the impact of the Younger Dryas", American Antiquity 57, pp. 482-494.

Moore, A.M.T., Hillman, G.C., Legge, A.J., 2000, Village on the Euphrates. From foraging to farming at Abu Hureyra. Oxford.

Mori, N., Ishii, T., Ishido, T., Hirosawa, S., Watatani, H., Kawahara, T., Nesbit, M., Belay, G., Takumi, S., Ogihara, Y., Nakamura, C., 2003, "Origins of domesticated emmer and common wheat inferred from chloroplast DNA fingerprinting", Proceedings of the 10th International Wheat Genetics Symposium, Paestum, pp. 25-28.

Nadel, D., 2002, Ohalo II. A 23,000-year-old fisher-hunter-gatherers' camp on the shore of the Sea of Galilee. Haifa.

Nadel, D., Danin, A., Power, R.C., Rosen, A.M., Bocquentin, F., Tsatskin, A., Rosenberg, D., Yeshurun, R., Weissbrod, L., Rebollo, N.R., Barzilai, O., Boaretto, E., 2013, "Earliest floral grave lining from 13,700-11,700-y-old Natufian burials at Raqefet Cave, Mt. Carmel, Israel", Proceedings of the National Academy of Sciences 110, pp. 11774-11778.

Özkan, H., Brandolini, A., Schäfer-Pregl, R., Salamini, F., 2002, “AFLP analysis of a collection of tetraploid wheats indicates the origin of emmer and hard wheat domestication in Southeast Turkey", Molecular Biology and Evolution 19, pp. 1797-1801.

Özkan, H., Willcox, G., Graner, A., Salamini, F., Kilian, B., 2011, "Geographic distribution and domestication of wild emmer wheat (Triticum dicoccoides)", Genetic Resources and Crop Evolution 58 (1), pp 11-53.

Pasternak, R., 1998, "Investigations of botanical remains from Nevali Çori, PPNB, Turkey: a short interim report”, in: A.B. Damania, J., Valkoun, G., Willcox, C.O., Qualset (eds.), The origins of agriculture and crop domestication, Aleppo, pp. 170-177.

Piperno, D.R., Weiss, E., Holst, I., Nadel, D., 2004, "Processing of wild cereal grains in the Upper Palaeolithic revealed by starch grain analysis", Nature 430, pp. 670-673.

Portillo, M., Kadowaki, S., Nishiaki, Y., Albert, R.M., 2014, "Early Neolithic household behavior at Tell Seker al-Aheimar (Upper Khabur, Syria): a comparison to ethnoarchaeological study of phytoliths and dung spherulites", Journal of Archaeological Science 42, pp. 107-118. 
Purugganan, M.D., Fuller, D.Q., 2011, “Archaeological data reveals slow rates of evolution during plant domestication”, Evolution 65 (1), pp. 71-183.

Ramsey, M.N., Maher, L.A., Macdonald, D.A., Nadel, D., Rosen, A.M., 2018, "Sheltered by reeds and settled on sedges: Construction and use of a twenty thousandyear-old hut according to phytolith analysis from Kharaneh IV, Jordan", Journal of Anthropological Archaeology 50, pp. 85-97.

Ramsey, M.N., Rosen, A.M., Nadel, D., 2017. Centered on the wetlands: integrating new phytolith evidence of plant-use from the 23,000-year-old site of Ohalo II, Israel", American Antiquity 82, pp. 702-722.

Riehl, S., Zeidi, M., Conard, N.J., 2013, "Emergence of agriculture in the foothills of the Zagros Mountains of Iran", Science 341, pp. 39-40.

Savard, M., Nesbitt, M., Jones, M.K., 2006, "The role of wild grasses in subsistence and sedentism: new evidence from the northern Fertile Crescent", World Archaeology 38, pp. 179-196.

Tanno, K., Willcox, G., 2006a, “How Fast Was Wild Wheat Domesticated?” Science 311, pp. 1886.

Tanno, K., Willcox, G., 2006b, "The origins of cultivation of Cicer arietinum L. and Vicia faba L.: early finds from Tell el-Kerkh, north-west Syria, late $10^{\text {th }}$ millennium B.P.", Vegetation History and Archaeobotany 15, pp. 197-204.

Tanno, K., Willcox, G., 2012, "Distinguishing wild and domestic wheat and barley spikelets from early Holocene sites in the Near East", Vegetation History and Archaeobotany 21, pp. 107-115.

Ucko, P.j., Dimbleby, G.W., 1969, The domestication and exploitation of plants and animals. London.

van Zeist, W., 1986, "Plant remains from Neolithic el Kowm, Central Syria", in R.H. Dornemann (ed.), A Neolithic village at tell el Kowm in the Syrian desert, Chicago, pp. 65-69.

van Zeist, W., Bakker-Heeres, J.A.H., 1982, “Archaeobotanical studies in the Levant. I. Neolithic sites in the Damascus Basin: Aswad, Ghoraifé, Ramad", Palaeohistoria 24, pp. 165-256.

van Zeist, W., Bakker-Heeres, J.A.H., 1984, “Archaeobotanical studies in the Levant.

2. Neolithic and Halaf levels at Ras Shamra”, Palaeohistoria 26, pp. 151-170.

van Zeist, W., de Roller, G.J., 1991/1992, “The plant husbandry of Aceramic Çayönü,

S.E. Turkey”, Palaeohistoria 33/34, pp. 65-96.

van Zeist, W., de Roller, G.J., 1995, "Plant remains from Asikli Höyük, a pre-pottery

Neolithic site in central Anatolia", Vegetation History and Archaeobotany 4, pp. 179-185.

van Zeist, W., de Roller, G.J., 2003, “The Cayönü archaeobotanical record”, in W. van Zeist (ed.), Reports on archaeobotanical studies in the Old World, Groningen, pp. 143-166.

Wallace, M., Jones, G., Charles, M., Forster, E., Stillman, E., Bonhomme, V., Livarda, A., Osborne, C.P., Rees, M., Frenck, G., Preece, C., 2018, "Re-analysis of archaeobotanical remains from pre- and early agricultural sites provides no evidence for a narrowing of the wild plant food spectrum during the origins of agriculture in southwest Asia", Vegetation History and Archaeobotany 28, pp. 449-463

Weide, A., Riehl, S., Zeidi, M., Conard, N.J., 2017, "Reconstructing subsistence practices: taphonomic constraints and the interpretation of wild plant remains at aceramic Neolithic Chogha Golan, Iran”, Vegetation History and Archaeobotany 26, pp. 487-504.

Weide, A., Riehl, S., Zeidi, M., Conard, N.J., 2018, "A systematic review of wild grass exploitation in relation to emerging cereal cultivation throughout the Epipalaeolithic and aceramic Neolithic of the Fertile Crescent", PLOS ONE 13, pp. e0189811. 
Weiss, E., 2017, "Palaeolithic Vegetal Diet in the Southern Levant", in O. Bar-Yosef y Y., Enzel (eds.), Quaternary of the Levant: Environments, Climate Change, and Humans. Cambridge, pp. 329-336.

Weiss, E., Kislev, M.E., Hartmann, A., 2006, "Autonomous cultivation before domestication", Science 312, pp. 1608-1610.

Weiss, E., Kislev, M.E., Simchoni, O., Nadel, D., 2004a, "Small-grained wild grasses as staple food at the 23,000 year old site of Ohalo II, Israel”, Economic Botany 58, pp.125134.

Weiss, E., Kislev, M.E., Simchoni, O., Nadel, D., Tschauner, H., 2008, "Plant-food preparation area on an Upper Paleolithic brush hut floor at Ohalo II, Israel", Journal of Archaeological Science 35, pp. 2400-2414.

Weiss, E., Wetterstrom, W., Nadel, D., Bar-Yosef, O., 2004b, “The broad spectrum revisited: Evidence from plant remains", Proceedings of the National Academy of Sciences of the United States of America 101, pp. 9551-9555.

Weiss, E., Zohary, D., 2011, "The Neolithic Southwest Asian founder crops: their biology and archaeobotany", Current Anthropology 52, pp. 237-254.

White, C.E., Makarewicz, C.A., 2012, "Harvesting practices and early Neolithic barley cultivation at el-Hemmeh, Jordan", Vegetation History and Archaeobotany 21, pp. 85-94.

Whitlam, J., Bogaard, A., Matthews, R., Matthews, W., Mohammadifar, Y., Ilkhani, H., Charles, M., 2018, "Pre-agricultural plant management in the uplands of the central Zagros: the archaeobotanical evidence from Sheikh-e Abad", Vegetation History and Archaeobotany 27, pp. 817-831.

Willcox, G., 2002, "Charred plant remains from a 10th millennium BP kitchen at Jerf el Ahmar (Syria)", Vegetation History and Archaeobotany 11, pp. 55-60.

Willcox, G., 2012, "The beginnings of cereal cultivation and domestication in Southwest Asia", in D.T. Potts (ed.), A companion to the archaeology of the ancient Near East, Oxford, pp. 163-180.

Willcox, G., 2013, "Food preparation and consumption on Late Pleistocene/Early Holocene sites in the Near East: Evidence from plant remains and archaeological finds", in L.Milano (ed.), Paleonutrition and food practices in the ancient Near East towards a multidisciplinary approach, Padova, pp. 1-10.

Willcox, G., Buxó, R., Herveux, L., 2009, "Late Pleistocene and Early Holocene climate and the beginnings of cultivation in northern Syria", The Holocene 19, pp. 151158.

Willcox, G., Fornite, S., Herveux, L., 2008, "Early Holocene cultivation before domestication in northern Syria", Vegetation History and Archaeobotany 17, pp. 313-325. 